\title{
Systèmes de Gestion des Connaissances pour la chaîne logistique intra- organisationnelle, Cas de la société BONFIGIOLI
}

\section{Knowledge Management Systems in intra-organizational Supply Chains: an Action-Research in BONFIGIOLI}

\author{
Cécile GAUMAND \\ Responsable Logistique BONFIGLIOLI France \\ Doctorante Laboratoire Génie Industriel \\ Knowledge Management Research Group \\ Ecole Centrale Paris \\ Grande Voie de Vignes \\ 92295 Châtenay-Malabry \\ gaumand@bonfiglioli.fr
}

Cécile Gaumand exerce depuis plus de quinze ans dans la fonction logistique. Actuellement responsable logistique chez Bonfiglioli Transmission, elle est doctorante à l'Ecole Centrale Paris dans continuité du master de recherche en logistique et systémique réalisé au CNAM.

\author{
Alain CHAPDANIEL \\ Coordinateur Executive Education \\ Laboratoire Génie Industriel \\ Chaire Supply Chain \\ Ecole Centrale Paris \\ Grande Voie de Vignes \\ 92295 Châtenay-Malabry \\ alain.chapdaniel@ecp.fr
}

Alain Chapdaniel est enseignant-chercheur à l'Ecole Centrale Paris, responsable formation continue de la chaire Supply chain de l'Ecole Centrale Paris, consultant, formateur dans la supply chain, les achats, les systèmes d'information pour le commerce électronique.

Aurélie DUDEZERT

Maître de Conférences- HDR

Laboratoire Génie Industriel

Knowledge Management Research Group

Ecole Centrale Paris

Grande Voie de Vignes

92295 Châtenay-Malabry

aurelie.dudezert@ecp.fr

Aurélie Dudezert est maître de conférences en sciences de gestion, habilitée à diriger les recherches, à l'École centrale de Paris et en charge du développement du Knowledge Management Research Group du Laboratoire Génie Industriel. 


\title{
Systèmes de Gestion des Connaissances pour la chaîne logistique intra- organisationnelle, Cas de la société BONFIGIOLI
}

\author{
Knowledge Management Systems in intra-organizational Supply Chains: \\ an Action-Research in BONFIGIOLI
}

\begin{abstract}
Résumé
L'optimisation de la chaîne logistique est devenue un des enjeux majeurs des entreprises ces dernières années. La Gestion des Connaissances peut être une des voies d'amélioration de la performance de la chaîne logistique. En tenant compte des spécificités des connaissances et de leur gestion dans la chaîne logistique, et en s'appuyant sur une recherche-intervention au sein du service logistique de Bonfiglioli Transmission, cet article propose un cadre de conception des Systèmes de Gestion des Connaissances pour la chaîne logistique intra-organisationnelle. Il met en évidence que dans ce contexte les Technologies de l'Information (TI) sont moins mobilisées en tant qu'outils de gestion des connaissances qu'en tant qu'outils de transformation des schémas cognitifs. Les TI jouent le rôle d'artefacts permettant de créer des interactions entre individus pour créer, stocker, partager et utiliser les connaissances organisationnelles.
\end{abstract}

Mots clefs :

Gestion des connaissances, chaîne logistique, Système de Gestion des Connaissances

\begin{abstract}
Optimisation of Supply Chain is today an issue for companies. This paper explores how Knowledge Management could improve the efficiency of Supply Chains. It investigates the specificities of knowledge of Supply Chain collaborators and the specificities of their management. With an Action Research methodology it proposes a framework and recommendations to design efficient Knowledge Management Systems for intra-organizational Supply Chains. It shows that in this context Information Technologies (TI) are rather used to change cognitive schemes than to manage knowledge. IT are used as artifacts that interact with individuals in creating, stocking, sharing and using organizational knowledge.
\end{abstract}

Keywords:

Knowledge Management, Supply Chain Management, Knowledge Management Systems 


\section{Introduction}

L'optimisation de la chaîne logistique (Supply Chain) est devenue un des enjeux majeurs des entreprises ces dernières années. Personnalisation croissante de l'offre de produits et services, exigences accrues en terme de délais, internationalisation des activités, environnement économique de plus en plus incertain, pression sur les résultats financiers : face à de tels enjeux une conception et gestion optimale de la chaîne logistique contribuent à l'efficacité, à la compétitivité et à la flexibilité des entreprises, favorisant ainsi leur aptitude à la création de valeur (Chu, 2008).

La chaîne logistique est un réseau complexe, orienté de plus en plus vers la demande du client final. Elle implique l'entreprise et ses réseaux (fournisseurs, distributeurs, prestataires) pour concevoir et acheminer efficacement les produits ou services aux clients finaux, ces derniers faisant parfois partie intégrante de la chaîne logistique. Spalanzani (2003, p. 31) décrit ainsi le Supply Chain Management comme « la gestion des flux physiques et d'information du client du client au fournisseur du fournisseur, afin d'offrir une réponse la plus satisfaisante possible aux besoins des clients ». De fait le concept de chaîne logistique n'est pas réduit à la seule partie amont (entreprise-fournisseurs) de la filière. L'image qu'emploient certains auteurs à propos de cette structure organisationnelle est celle de « l'entreprise archipel» (Fabbe-Costes, 2008) qui doit gérer de multiples interfaces avec d'autres entreprises et dont la réussite individuelle est largement liée aux réactions, aux compétences et à la réussite des autres. Ainsi cette structure se caractérise par la mise en œuvre de processus transverses à l'entreprise (de la conception de produit/service à sa commercialisation) mais également par une très forte interaction des acteurs de la chaîne logistique avec l'environnement externe et les parties prenantes de l'entreprise.

L'enjeu pour les entreprises est de mettre en place des chaînes logistiques à la fois intégrées (synchronisation des activités et coordination des intervenants) et flexibles (adaptabilité des processus et capacité à adapter et à transformer les chaînes) pour faire face aux évolutions de l'environnement ou de la demande client (Fabbe-Costes, 2007). Cette optimisation peut être recherchée en premier lieu à un niveau interne à l'entreprise, autrement appelé niveau intra-organisationnel (Pagell, 2004). Ce niveau est considéré comme le premier stade de l'intégration logistique. Il suppose une intégration des 
fonctions internes à l'entreprise concernées par la gestion de la chaîne logistique (R\&D, marketing, production, distribution...). Il ouvre la voie à un deuxième stade de l'intégration logistique appelé niveau inter-organisationnel et incluant l'ensemble des partenaires de la chaîne et notamment les fournisseurs mais aussi clients.

Afin d'optimiser la gestion de cette chaîne logistique, l'accent a été mis depuis plusieurs années sur l'optimisation des flux physiques, informationnel et financier de cette chaîne. Des outils et méthodes de gestion de production et distribution (Kanban, commande horodatée sur prévision, approvisionnement synchrone sur commande ferme, gestion de tournée....) ou la mise en place de services Supply Chain professionnalisés ont notamment permis de réduire les coûts et les délais. En parallèle l'informatisation des données et l'apparition de technologies de Customer Relationship Management (CRM) ou de gestion de données (ERP) ont facilité la gestion des flux d'information (transmission des sorties de caisses de magasins au fournisseur, utilisation de RFID pour tracer les produits et faciliter leur gestion...). Aujourd'hui d'autres moyens d'optimisation sont recherchés. Quelques pratiques d'amélioration de la gestion de la chaîne logistique grâce à la Gestion des Connaissances comme celle de WAL MART (Binot et Dudezert, 2008) ont conduit récemment le monde académique à s'intéresser plus précisément à cette voie d'amélioration de la performance de la chaîne logistique. Toutefois comme le soulignent de nombreux auteurs, les recherches dans ce domaine sont aujourd'hui encore émergentes et peu nombreuses (Lancini, 2007 ; Gunasekaran et Ngai, 2007) et traitent principalement de l'optimisation de la chaîne logistique inter-organisationnelle (Evrard Samuel et Spalanzani, 2009 ; Fabbe-Costes et Lancini, 2009 ; Hult et ali, 2003 ; 2006). Audelà, les spécificités de la chaîne logistique font que les modes traditionnels de Gestion des Connaissances élaborés autour de Systèmes de Gestion des Connaissances ne répondent que partiellement à ses besoins.

Aussi, en tenant compte des spécificités des connaissances et de leur gestion dans la chaîne logistique et au travers d'une recherche-intervention au sein du système logistique de l'entreprise BONFIGIOLI, cet article propose un cadre de conception de Systèmes de Gestion des Connaissances pour la chaîne logistique intra-organisationnelle. Dans une première partie l'article discute le problème de la gestion des connaissances et de la conception de Systèmes de Gestion des Connaissances pour la chaîne 
logistique intra-organisationnelle au travers d'une analyse de la littérature. La deuxième partie présente la méthodologie de la recherche et le contexte de son application. Dans une troisième partie, nous présentons la recherche-intervention et ses résultats. Enfin une dernière partie discute les implications et les limites de ce travail.

\section{Chaîne logistique intra-organisationnelle et Gestion des}

\section{Connaissances}

\subsection{Les Systèmes de Gestion des Connaissances et leur mise en place}

La Gestion des Connaissances peut être définie comme « la génération, la représentation, le stockage, le transfert, la transformation, l'application, l'enracinement et la protection de connaissances organisationnelles. Mémoire organisationnelle, partage d'informations et travail collaboratif sont aussi étroitement liés à la notion de Gestion des Connaissances »(Schultze et Leidner, 2002). Cette Gestion des Connaissances peut prendre des formes organisationnelles très variées allant de la mise en place de bases de connaissances (Knowledge Bases) à la valorisation de connaissances formalisées comme les marques ou les brevets en passant par l'aménagement d'espaces de travail permettant l'échange de connaissances (Earl, 2001). Toutefois, ces démarches sont très souvent mises en œuvre au travers du déploiement de Systèmes d'Information spécifiques que sont les Systèmes de Gestion des Connaissances (SGC).

Ces SGC sont définis comme « des classes de Systèmes d'Information dédiés au management de la connaissance organisationnelle. C'est-à-dire qu'il s'agit de systèmes fondés sur des technologies de l'information développées pour supporter et améliorer les processus de création, stockage, recherche et identification, transfert et intégration de connaissances » (Alavi et Leidner, 2001). L'efficacité de leur mise en place doit prendre en compte le regard porté sur les connaissances par l'organisation, les objectifs opérationnels des processus impactés, comme les modèles d'affaires des organisations qui les mettent en oeuvre (Hansen et ali, 1999 ; Earl, 2001 ; Dudezert et Lancini, 2006). Ainsi les modèles d'audit ou de conception de Systèmes de Gestion des Connaissances (Aviv et ali, 2008 ; Dudezert et Lancini, 2006) considèrent l'ensemble de ces éléments comme déterminant pour 
qualifier/concevoir le Système de Gestion des Connaissances adéquat à l'organisation. Toutefois, ces modèles ont été construits pour concevoir des Systèmes de Gestion des Connaissances sur le périmètre d'une seule entité (un département ou un service) ou en considérant l'entreprise comme une entité n'ayant qu'un seul point de vue sur ses objectifs et sur la manière dont elle considère les connaissances. Dans le cas de la chaîne logistique intra-organisationnelle, ces cadres de conception ne ne permettent pas de développer des systèmes adéquats. Effectivement, la chaîne logistique traverse plusieurs services. Les objectifs opérationnels et le regard porté sur les connaissances sont alors différents. Un SGC adapté à la chaîne logistique devra prendre en compte ces différences. C'est pourquoi, la définition d'un cadre de conception de Systèmes de Gestion des Connaissances adapté à la chaîne logistique et prenant en compte ses spécificités est en soit un problème académique comme managérial.

\subsection{La spécificité des connaissances logistiques et de la gestion des connaissances de la chaîne logistique}

Les métiers de la chaîne logistique sont très variés et mobilisent des savoirs et compétences qui sont eux-mêmes d'une grande variété et comme le souligne Fabbe-Costes (2008) à caractère essentiellement « humains ». En traversant les services et départements de l'entreprise, les savoirs de la chaîne logistique sont certes techniques pour une partie d'entre eux mais surtout organisationnels, relationnels car situés aux interfaces, tacites avec une forte composante liée à l'expérience des individus et à leur savoir-faire. Ainsi pour Mathe et Tixier (1981, p.34) les logisticiens sont « des hommes d'interfaces chargés d'une mission de coordination dans le cadre d'une analyse globale ». Gammelgaard et Larson (2001) dans leur travail d'identification des compétences clefs des logisticiens rapportent que la littérature considère le logisticien comme un homme de coordination mais également comme un homme de « situation » et plus précisément comme un individu capable de s'adapter à des situations «spéciales » (Sheffi et Klaus 1997). La compétence du logisticien reposerait donc sur le développement d'un savoir d'action et de situation.

Plus précisément, pour beaucoup d'entre eux, les métiers de la chaîne logistique ne se sont professionnalisés, et leur importance stratégique n'a été reconnue, que très récemment. Aussi la grande majorité des acteurs de cette chaîne sont-ils encore des autodidactes peu enclins à la formalisation de 
leur connaissance métier. Au-delà, la pénibilité du travail, les rémunérations basses, la faible reconnaissance des fonctions opérationnelles poussent beaucoup de logisticiens à changer d'entreprise régulièrement (Camman \& Livolsi, 2007). La connaissance des logisticiens est de ce fait particulièrement difficile à maîtriser.

Enfin, les acteurs de la chaîne logistique sont des hommes et des femmes d'interfaces intraorganisationnelles mais également inter-organisationnels. Au cœur de la même entreprise, les acteurs de la chaîne logistique travaillent aux interfaces de la conception, de la production et de la distribution des produits et services. Bien plus, le département Logistique est au cœur des relations avec les fournisseurs, les prestataires et les clients. Cet accès à des informations externes à l'organisation comme la capacité à confronter leur propre savoir à ceux d'acteurs d'autres organisations inscrit les logisticiens dans un processus d'intégration des connaissances à l'entreprise et contribue fortement au renouvellement des connaissances et compétences de l'entreprise selon les processus évoqués par Caron-Fasan et Farastier (2003).

Ainsi les connaissances des acteurs de la chaîne logistique sont essentiellement des savoirs tacites, d'interfaces, de coordination, de situation et d'action, difficiles à maîtriser par l'entreprise. Toutefois ces connaissances sont des connaissances clefs pour l'entreprise au sens de la KnowledgeBased View (Kogut et Zander, 1992) car elles permettent non seulement une coordination et une synchronisation effective de l'ensemble des fonctions de l'entreprise (intégration de la chaîne logistique) mais contribuent également au renouvellement des connaissances internes de l'entreprise. De la sorte, le développement de ces connaissances et les processus d'apprentissage mis en œuvre dans la chaîne logistique sont susceptibles de contribuer à sa transformation et à son adaptabilité. Aussi, optimiser la gestion des connaissances de la chaîne logistique intra-organisationnelle est une voie à la fois pour améliorer le niveau d'intégration mais aussi pour améliorer le niveau de flexibilité et d'adaptabilité de la chaîne logistique. L'enjeu est alors de concevoir des démarches de gestion des connaissances pour la chaîne logistique intra-organisationnelle qui permettent de prendre en compte l'ensemble de leurs spécificités.

Il n'existe que peu de pratiques de Gestion des Connaissances de la chaîne logistique aujourd'hui. Concernant spécifiquement les démarches de GC pour la chaîne logistique intra- 
organisationnelle, Oppong et ali (2005) indiquent qu'elles semblent aujourd'hui essentiellement structurées autour d'outils de gestion de l'information visant à coordonner et stocker les connaissances formalisées au sein de l'entreprise. Ces démarches sont intéressantes et ont donné lieu dans les années 2000 à plusieurs expériences dans l'industrie. Toutefois comme le met en évidence Spalanzani (2003) ces démarches participent à une optimisation de la chaîne logistique amont (amélioration de la qualité dans les processus internes à l'entreprise) mais ne tirent que très partiellement partie des spécificités et de l'intérêt des connaissances des acteurs de la chaîne logistique.

Les Systèmes de Gestion des Connaissances adaptés à l'optimisation de la chaîne logistique intra-organisationnelle doivent gérer des savoirs tacites, d'interfaces, de coordination, de situation et d'action et ce dans un objectif d'amélioration de l'intégration et de la flexibilité de la chaîne logistique. Au niveau intra-organisationnel, en s'inspirant de la liste des facteurs facilitant cette intégration et adaptabilité de la chaîne logistique identifiée par Fabbe-Costes (2007), on peut ainsi considérer que le SGC doit contribuer :

- à identifier et sécuriser les points internes de la circulation des flux informationnels sensibles aux changements externes comme à choisir des formats de flux compatibles avec des standards inter-organisationnels ;

- à développer un savoir-faire en matière de modélisation, simulation, reconfiguration des processus comme de mesure et de pilotage de la performance des processus ;

- à faire des choix de standards internes en matière de technologies de l'information compatibles avec des standards inter-organisationnels ;

- à insérer les individus dans des réseaux sociaux larges ; à leur faire développer une vision des chaînes, des réseaux ; à faire émerger des leaders ; à développer la culture du projet, du risque, du changement et de l'innovation.

On identifie ici à la fois des objectifs de développement des connaissances spécifiques aux besoins de la chaîne logistique mais aussi des contraintes posées aux technologies supports du SGC. Le tableau 1 synthétise les spécificités des connaissances de la chaîne logistique, les objectifs de développement des connaissances susceptibles d'améliorer la performance de la chaîne logistique et les contraintes imposées aux Technologies de l'Information supports au SGC. 
Tableau 1. Spécificités propres aux SGC de la chaîne logistique intra-organisationnelle

\begin{tabular}{|l|l|l|}
\hline \multicolumn{1}{|c|}{$\begin{array}{c}\text { Spécificités des } \\
\text { connaissances }\end{array}$} & \multicolumn{1}{c|}{$\begin{array}{c}\text { Objectifs de développement des } \\
\text { connaissances pour la chaîne logistique }\end{array}$} & $\begin{array}{c}\text { Attendus des TI supports au } \\
\text { SGC }\end{array}$ \\
\hline $\begin{array}{l}\text { Savoirs tacites, } \\
\text { d'interfaces, de } \\
\text { coordination, de } \\
\text { situation et d'action }\end{array}$ & $\begin{array}{l}\text { - Objectif de développement des } \\
\text { connaissances « processus }: \\
\text { Développer des connaissances en } \\
\text { matière de modélisation, simulation, } \\
\text { reconfiguration des processus et en } \\
\text { matière de mesure et pilotage de la } \\
\text { performance des processus }\end{array}$ & $\begin{array}{l}\text { - Capacité à identifier et } \\
\text { sécuriser les points internes de } \\
\text { la circulation des flux } \\
\text { informationnels sensibles aux } \\
\text { changements externes } \\
\text { - Objectif de transformation des schémas } \\
\text { cognitifs: } \\
\text { Développer une culture de partage des de formats de flux et de } \\
\text { technologies compatibles avec } \\
\text { des standards inter- } \\
\text { organisationnels }\end{array}$ \\
& $\begin{array}{l}\text { connaissances, des réseaux et des chaînes, } \\
\text { du projet, du risque, du changement et de } \\
\text { l'innovation }\end{array}$ & \\
\hline
\end{tabular}

Proposer un SGC capable de répondre à l'ensemble de ces spécificités est un véritable enjeu en matière de gestion des connaissances. De fait, remplir l'objectif de développement des connaissances «processus » nécessite de mettre en place un processus spécifique d'explicitation et de formalisation des connaissances des acteurs de la chaîne logistique. Comme nous l'avons souligné plus haut, ces connaissances sont tout particulièrement tacites. Or pour remplir ce premier objectif le SGC doit tout à la fois permettre une explicitation et une formalisation des connaissances susceptibles de soutenir le développement de connaissances «processus » en interne à l'organisation, mais il doit également viser la compatibilité d'une telle explicitation et formalisation pour l'échange avec d'autres organisations. La question qui est posée est alors de savoir comment passer d'un savoir tacite lié à l'action à un savoir partagé au niveau intra-organisationnel et compatible avec un savoir partagé au niveau inter-organisationnel. Le lien de la chaîne logistique avec d'autres organisations externes fait que pour satisfaire cet objectif de développement des connaissances «processus » en interne à l'organisation, il ne s'agit pas uniquement d'un enjeu d'explicitation, de formalisation et de combinaison de connaissances en tenant compte d'un contexte culturel et intra-organisationnel spécifique et partagé (Bâ) au sens de Nonaka et Takeuchi (1995). Il s'agit plutôt dans ce cas d'un enjeu d'explicitation, formalisation et combinaison des connaissances intra-organisationnelles susceptible de donner lieu à une externalisation de ces connaissances qui pourrait elle-même 
contribuer à une dynamique de développement des connaissances inter-organisationnelles. Pour atteindre cet objectif, une voie intéressante semble tout à la fois de construire un SGC en tenant compte des spécificités des connaissances des acteurs de la chaîne logistique, des spécificités internes à l'organisation et du contexte partagé intra-organisationnel $(B \hat{a})$ mais aussi de se référer aux pratiques et aux savoirs partagés dans le domaine de la logistique (technologies mobilisées par la communauté des acteurs, référentiels métiers...), à savoir le $B a ̂$ inter-organisationnel.

La nécessité pour le SGC de la chaîne logistique intra-organisationnelle de remplir également un objectif de transformation des schémas cognitifs en vue de développer une culture du partage et des réseaux est une seconde originalité en matière de gestion des connaissances. De fait, les savoirs des acteurs de la chaîne logistique sont des savoirs de situation, d'urgence et d'action. La gestion des activités et la résolution des problèmes se fait dans l'urgence et à un niveau individuel. L'enjeu est alors de transformer les schémas cognitifs à deux niveaux. Tout d'abord faire comprendre que la résolution de problèmes se fait au niveau individuel mais que cette résolution peut être améliorée si l'acteur ne vise plus seulement l'amélioration de sa tâche mais tient compte d'une logique intraorganisationnelle voire également d'une logique inter-organisationnelle (culture « réseaux »). Ensuite faire comprendre que le temps passé à l'amélioration de pratiques au niveau organisationnel (culture du partage des connaissances) n'est pas un temps perdu pour la résolution de tâches individuelles. Le SGC doit donc contribuer à une transformation profonde de la culture de travail et du contexte partagé par les acteurs de la chaîne logistique conduisant à l'agilité et à l'adaptabilité de la chaîne logistique (transformation du Bâ).

Au regard de ces spécificités, nous avons choisi de mener une recherche-intervention au sein de la chaine logistique BONFIGIOLI Transmission afin de proposer un cadre de conception des SGC pour la chaîne logistique intra-organisationnelle. 


\section{Le choix d'une recherche-intervention au sein d'une entreprise en transformation}

\subsection{Méthodologie de recherche}

Sur ces sujets de recherche émergents où la compréhension de la complexité organisationnelle parât nécessaire pour traiter le problème, la recherche-action (Baskerville, 1999) ou sa variante plus ingénierique la recherche-intervention (David, 2000) paraît une méthode particulièrement adaptée. Ces méthodologies de recherche ont pour objectif tout à la fois de résoudre un problème opérationnel (création de connaissances pour l'action) mais également d'enrichir le corpus de connaissances scientifiques sur ces problèmes organisationnels. Elles partent du principe que les organisations humaines ne peuvent être comprises qu'en adoptant une vision globale et que la meilleure manière de comprendre ces organisations est d'introduire des changements et d'observer l'effet de ces changements. Elles conduisent à une implication des acteurs de l'entreprise dans le projet de transformation de l'entreprise.

Comme le souligne David (2000), ces méthodes de recherche reposent sur quatre principes :

- un objectif de compréhension en profondeur du fonctionnement du système, associé à une démarche d'investigation particulière conduisant le chercheur à utiliser sa position pour co-produire des connaissances depuis l'intérieur du système et non depuis l'extérieur ;

- la production de connaissances se fait dans l'interaction avec le terrain. Elle se fait notamment via la conception et la mise en œuvre d'outils de gestion et de procédures ;

- le chercheur parcourt différents niveaux théoriques le conduisant notamment à revisiter sans cesse les théories existantes grâce aux matériaux empiriques. Ceci nécessite notamment pour le chercheur de maitriser les concepts de la théorie des organisations, de connaître les outils de gestion mais également de savoir acquérir les compétences techniques ;

- l'intervention a naturellement un caractère normatif. 
Deux phases de travail structurent la démarche du chercheur qui adopte ces méthodologies (Baskerville, 1999) :

- une phase de diagnostic qui implique une analyse du contexte de l'organisation étudiée en collaboration avec les acteurs de l'entreprise

- une phase dite «thérapeutique » dans laquelle le chercheur introduit des éléments de transformation de l'organisation pour étudier leurs impacts.

Compte tenu du domaine de notre recherche et du contexte de l'entreprise Bonfiglioli décrits ci-après, la méthode de recherche-intervention nous est apparue tout particulièrement adaptée à notre recherche. Il s'agit pour nous d'étudier un sujet émergent (peu de travaux académiques) pour explorer l'impact des spécificités contextuelles de la chaîne logistique intra-organisationnelle sur les Systèmes de Gestion des Connaissances à développer. Par ailleurs, notre position nous amène également à apporter des solutions opérationnelles à l'optimisation de la chaîne logistique via la Gestion des Connaissances. Pour reprendre l'expression de David (2000, p. 210), l'objectif de notre recherche est tout à la fois de faire du terrain « un lieu d'ingénierie (conception de modèles et d'outils de gestion adéquats, y compris modèles et outils de pilotage du changement) et source de théories fondées (ce que la conception et la mise en place de ces outils révèlent sur le fonctionnement des organisations, et qui vient enrichir le corpus des connaissances théoriques en sciences de gestion)».

La recherche-intervention présentée dans cet article s'est déroulée de Février 2008 à Février 2009 au sein de la société Bonfiglioli. L'équipe en charge du projet de recherche est composée de trois chercheurs :

- une doctorante ayant une expérience d'une quinzaine d'années dans le domaine du management de la logistique au sein de différentes entreprises industrielles. Cette jeune chercheuse a été embauchée en CDI à partir de Février 2008 au sein de la branche française de Bonfiglioli pour tenir la fonction de Responsable de la fonction Supply Chain. C'est elle qui a opérationnalisé l'intervention. 
- Un chercheur-associé ayant une expérience d'une vingtaine d'années dans le management de la logistique et la formation des managers logistiques. Ce chercheur est aussi en charge de la coordination de la formation continue au sein d'une chaire en Supply Chain ayant pour objectif de faire progresser le management de la chaîne logistique en partenariat avec six grandes entreprises.

- Une enseignante-chercheur spécialisée sur les sujets de Gestion de la Connaissance, responsable de l'encadrement scientifique de la recherche.

Mener cette recherche-intervention avec cette équipe présente deux atouts :

- Créer un climat de confiance avec l'entreprise permettant de mener l'expérimentation en toute sérénité. Introduire la GC dans la chaîne logistique intra-organisationnelle est aujourd'hui encore une pratique innovante. Aussi l'entreprise peut être craintive vis-à-vis d'une telle expérimentation. La présence d'une chercheuse-intervenante ayant une quinzaine d'années d'expérience en management de la chaîne logistique, associé à la présence d'un expert en management de la chaîne logistique travaillant avec un réseau de grandes entreprises dans les domaines de la chaîne logistique permet de rassurer l'entreprise.

- Pouvoir mener une expérimentation à un niveau hiérarchique suffisamment élevé pour mener l'étude sur l'ensemble de la chaîne logistique intra-organisationnelle. Le fait d'être positionné à la tête du Département Logistique et d'avoir le soutien de la Direction Générale pour une telle expérimentation est un point fort de la recherche.

Seule la doctorante a opérationnalisé l'intervention. Par le biais d'échanges mensuels entre les trois membres de l'équipe, des orientations ont été données pour améliorer l'intervention et la cadrer au regard de la question de recherche à explorer.

\subsection{Bonfiglioli : Histoire ; activité ; culture}

Bonfiglioli, entreprise familiale, est devenue en moins de 50 ans une organisation multinationale constituée de sites de production et de filiales commerciales dans plus de 14 pays, avec plus de 2600 collaborateurs. Sa croissance annuelle sur les 5 dernières années est de $20 \%$ par an. 
La mémoire collective du groupe est incarnée par l'image et l'esprit visionnaire du fondateur. Clementino Bonfiglioli fonda l'entreprise en 1956 afin de répondre à la demande en pièces de rechange des constructeurs de machines agricoles sur la région bolognaise. En quelques années il recentra son cœur d'activité sur la conception, fabrication et commercialisation de moto-réducteurs pour le secteur industriel. L'objectif étant, à ce moment, de répondre à la demande croissante des industriels sur la zone de Bologne. La filiale française, Bonfiglioli Transmission, (qui est notre périmètre d'expérimentation) existe depuis 30 ans et s'est appropriée la culture familiale du groupe où son fondateur, à 80 ans, est toujours en fonction et s'est entouré de ses enfants pour la gestion du groupe. Les méthodes originelles de gestion des processus d'entreprise perdurent chez Bonfiglioli Transmission. Notamment la forte implication des fonctions commerciales dans les processus achats, approvisionnements et même gestion de la production (y compris lancement d'ordre de fabrication avec leurs spécifications techniques).

La fonction logistique de Bonfiglioli Transmission représente une trentaine de personnes réparties sur six grandes fonctions : approvisionnement, gestion des stocks, ordonnancement, réception, préparation de commandes, et expédition, avec comme particularité de comprendre la production. Ce département logistique n'a que 3 ans d'existence. En effet, historiquement les services cités ci-dessus étaient sous la hiérarchie directe du Directeur Général. Selon la culture organisationnelle, les commerciaux donnaient des instructions aux logisticiens, aussi bien en matière d'approvisionnement, de réception, de production, que d'expédition. La combinaison des deux fonctions commerciale et logistique a généré le développement d'une certaine agilité face à la gestion des priorités commerciales. Effectivement les acteurs de la fonction logistique recevant des ordres directement des commerciaux sur demande des clients, ont développé une forte capacité de compréhension et d'interprétation du besoin client tant sur le produit à livrer que sur le délai à tenir. Cette agilité et forte capacité d'adaptation des acteurs de la fonction logistique s'est développée également en réponse à la nécessité de gérer des aléas quotidiens aussi bien en interne qu'en externe au système logistique (retards des transporteurs, retards fournisseurs, problèmes qualités produits, etc.). 
La fonction logistique de Bonfiglioli Transmission est représentative de l'autodidactie des logisticiens puisque $6 \%$ des logisticiens possèdent une formation logistique. De plus, la forte rotation de logisticiens opérant dans les fonctions opérationnelles en réception et préparation rend plus difficile le maintien du niveau des connaissances et des pratiques organisationnelles. Le système logistique Bonfiglioli Transmission entretient également des échanges de connaissances avec son environnement (essentiellement avec ses principaux fournisseurs). Ces échanges portent principalement sur des prévisions de ventes et d'évolutions techniques. Toutefois ces échanges ne sont que très peu intégrés avec les connaissances clients et marchés détenues par les commerciaux de l'entreprise.

La stratégie du groupe Bonfiglioli depuis 2008 est de passer d'une offre produits à une offre solution intégrant plusieurs lignes de produits et services, afin d'atteindre l'excellence sur les marchés de référence. Cette stratégie s'appuie sur les leviers d'action suivants:

(1) Une réorganisation du groupe en divisions commerciales (business unit).

(2) Des investissements (10\% du CA en 5 ans) alloués pour robotiser la production italienne, pour créer des sites sur les continents européen, américain, et asiatique, ainsi que pour la recherche et l'innovation dans le domaine de la mécatronique

(3) Une consolidation des sites et filiales pour permettre une meilleure intégration et harmonisation des structures de données, des processus de gestion de la demande et de gestion des ressources de la Supply Chain Bonfiglioli. (mise en place d'un ERP, CRM, Certification Groupe ISO)

(4) Une meilleure gestion des connaissances et des talents permettant d'atteindre l'excellence.

Bonfiglioli Transmission représente un acteur majeur dans le déploiement de cette stratégie et se retrouve impliqué (objectifs 1, 2,3) et pilote pour le déploiement de l'objectif 4. La filiale française est la première à déployer l'ERP et fait partie des premières filiales à intégrer la démarche d'une certification ISO9001 Groupe. A ce premier stade nous mettons en évidence la volonté du groupe de s'inscrire dans une démarche d'intégration des processus entre usines et filiales. Ceci apporte une dynamique de changement sur laquelle nous nous appuyons dans notre démarche. 


\section{L'intervention au sein de Bonfiglioli Transmission}

\subsection{Phase de diagnostic}

La phase de diagnostic se compose essentiellement de deux audits menés en parallèle : le repérage des connaissances logistiques et l'évaluation de la maturité du système logistique.

\subsubsection{Repérer les connaissances logistiques}

Comme le souligne la littérature, le système logistique mobilise des connaissances très variées. Aussi une des premières actions fut de dresser un état des lieux des connaissances détenues par les acteurs de la fonction logistique de Bonfiglioli Transmission. Cette phase de repérage est aussi une première phase d'explicitation des connaissances des acteurs ouvrant la voie à l'objectif de formalisation des connaissances que doit remplir le SGC. Pour cela nous avons réalisé une cartographie des connaissances de chaque membre de la fonction logistique. Les cartographies de connaissances permettent d'expliciter les connaissances via la parole et la visualisation (Dudezert, 2007). Ceci semblait bien adapté pour une population peu familière avec la culture de l'écrit. Pour aider à la réalisation de cette cartographie, une pré-cartographie des connaissances logistiques (voir figure 1) a été réalisée en amont des entretiens en se fondant sur la norme AFNOR NF X 50-600 et la littérature. En s'appuyant sur un référentiel métier partagé par la communauté des acteurs des métiers de la logistique dès cette étape, l'objectif était aussi de prendre en compte les visées de compatibilité de l'explicitation des connaissances avec d'autres organisations évoquées plus haut.

En tant que Responsable Logistique nouvellement recrutée, nous avons proposé aux principaux acteurs de la fonction logistique des entretiens individuels afin de mieux comprendre la nature de leur métier au quotidien. Ainsi nous avons réalisé 13 entretiens sur un mois. Chacun de ces entretiens individuels duraient entre $1 \mathrm{~h} 30$ et $3 \mathrm{~h}$. Durant cet échange une cartographie des connaissances du professionnel logistique était dressée à partir de la pré-cartographie des connaissances logistiques représentant de façon visuelle les connaissances techniques, fonctionnelles et humaines des acteurs de la fonction logistique. Lors des entretiens, la pré-cartographie était discutée et modifiée de façon à créer une nouvelle cartographie représentative des connaissances développées et utilisées lors de l'activité quotidienne du collaborateur interviewé. 


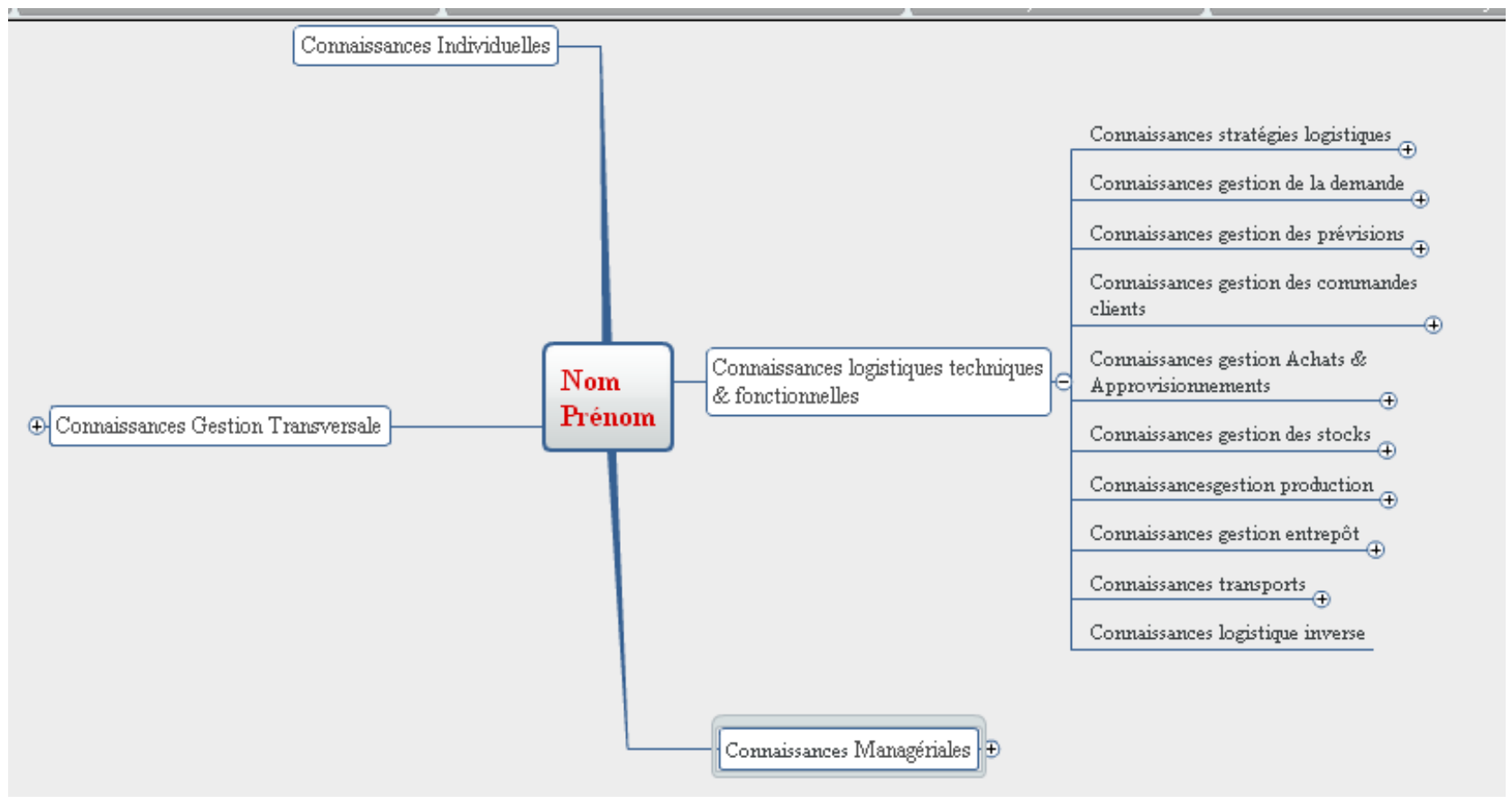

Figure 1. La pré-cartographie des connaissances de la fonction logistique Bonfiglioli

La réalisation de ces cartographies de connaissances a permis de mettre en évidence la forte implication de la fonction commerciale dans la fonction logistique. Il nous est alors apparu important de mieux comprendre le fonctionnement et les incidences de cette interface entre le service logistique et le service commercial. C'est pourquoi nous avons complété le travail de cartographie par un second travail d'analyse des influences entre acteurs. Pour cela nous nous sommes appuyés sur la méthode MACTOR (Godet, 2006). La méthode MACTOR (Méthode ACTeurs, Objectifs, Rapports de force) cherche à estimer les rapports de force entre acteurs et à étudier leurs convergences et divergences visà-vis d'un certain nombre d'enjeux et d'objectifs associés. Nous avons utilisé cette méthode avec quatre acteurs que nous avions identifiés préalablement comme étant les quatre piliers du service logistique (le responsable production, le responsable expédition, le responsable réception et le responsable approvisionnement). En déployant la méthode et en créant avec eux les cartographies d'acteurs fondés sur leurs objectifs, leurs projets en cours, leurs motivations, leurs contraintes, leurs moyens d'action internes, nous avons précisé la nature des réseaux d'influence présents dans la fonction logistique. Ceci nous a permis de préciser les enjeux de pouvoir entre acteurs susceptibles 
d'avoir un impact sur le développement et la gestion des connaissances de la chaîne logistique de Bonfiglioli Transmission. Ceci s'est avéré utile lors de la phase thérapeutique de l'intervention.

\subsubsection{Evaluer la maturité du système logistique}

Dès notre arrivée chez Bonfiglioli nous avons recherché toutes les documentations décrivant le système logistique et ses processus. La conclusion de cette analyse documentaire confirma le manque d'utilisation de méthodes logistiques (MRP, politique d'approvisionnement, stratégie d'achat ou de fabrication, etc.) et mis en évidence l'interventionnisme de la fonction commerciale dans le système logistique (requête d'achat, demande de stock de sécurité émises sur la seule demande commerciale).

Pour compléter cette analyse documentaire, nous avons évalué la maturité du système logistique en utilisant le référentiel ASLOG (Evaluation de l'aptitude à la performance logistique (E4/2005). Le référentiel ASLOG (Association française pour la logistique créée en 1972) comme le référentiel SCOR (crée par le Supply Chain Council en 1996) part d'une modélisation du système logistique global pour ensuite décrire les sous-processus et moyens de contrôle. Nous avons choisi ces référentiels car ils sont les plus reconnus de la profession et en sont devenus les standards. Toutes les entreprises et acteurs de la chaîne logistique sont aujourd'hui familiers de ces standards.

A l'issue de cette évaluation, il s'avère que $45 \%$ des critères démontrent une absence du minimum requis pour l'obtention du niveau 1 du standard ASLOG. Sur les 13 domaines audités par le référentiel ASLOG, 6 sont concernés (approvisionnement, maitrise des flux d'information, conduite du progrès, gestion des indicateurs de pilotage, management stratégique, gestion des ressources humaines). Bien plus, seuls $45 \%$ des critères possèdent le niveau 1 nécessaire pour passer au niveau 2.

Cette phase d'audit nous donna suffisamment de matière pour créer notre plan d'action et notre phase thérapeutique.

\subsection{Phase thérapeutique}

Suite à l'audit interne logistique présenté ci-dessus nous avons identifié qu'effectivement la fonction logistique chez Bonfiglioli mobilisait des connaissances très nombreuses et variées. Nous avons également identifié que le niveau de maturité de la chaîne logistique en matière d'intégration et 
de flexibilité était relativement faible. Dans ce contexte, chercher à améliorer cette intégration et flexibilité par la mise en place d'un Système de Gestion des Connaissances paraissait pertinent. Comme nous l'avons déjà souligné, deux objectifs de développement des connaissances semblent plus particulièrement adaptés à la chaîne logistique : un objectif de développement des connaissances «processus » et un objectif de transformation des schémas cognitifs pour développer une culture des réseaux, des chaînes, du projet, du risque, du changement et de l'innovation. Nous décrivons ci-après les démarches mis en œuvre chez Bonfiglioli pour atteindre ces deux objectifs.

\subsubsection{Développer les connaissances en matière de modélisation, simulation, reconfiguration, mesure et pilotage des processus}

Pour atteindre cet objectif, nous nous sommes rattachés à un des leviers de la mise en œuvre de la stratégie de Bonfiglioli : la mise en place d'un ERP. Les ERP visent à optimiser les processus de gestion des entreprises en proposant un référentiel unique s'appuyant sur des règles de gestion standardisées (Reix, 2005). Installer et utiliser un ERP entraîne une standardisation et une intégration des processus de l'entreprise. Toutefois, l'implantation d'un ERP ne se révèle pas seulement un exercice technologique mais aussi une révolution organisationnelle et esquisse les prémisses d'un apprentissage collectif (Akkermans \& van Helden, 2002; Wei et ali, 2005). Plus précisément, l'implémentation d'un ERP va naturellement conduire à expliciter, partager puis combiner la connaissance que chaque acteur possède sur les processus et les modèles de fonctionnement de l'organisation (Jones et ali, 2006). Bien plus, comme le souligne El Amrani (2008, p. 63), la mise en place d'un ERP peut contribuer au développement d'une vision transversale c'est-à-dire contribuer à « la perception qu'ont les acteurs de l'entreprise de la nature intégrée et couplée des différents processus organisationnels et leur capacité à comprendre les différentes interactions qu'ils ont avec le reste de l'organisation ». Enfin, les ERP représentent l'un des éléments le plus important du système d'information et de communication du système logistique. Ces technologies sont largement déployées dans les chaînes logistiques des entreprises et leur utilisation fait partie de pratiques désormais partagées entre les acteurs de la communauté des métiers de la logistique.

Aussi s'appuyer sur la mise en place d'un ERP nous est apparu à la fois comme une opportunité de développer les connaissances «processus», leur explicitation, leur partage et leur 
combinaison intra-organisationnelle avec une logique de compatibilité inter-organisationnelle mais aussi comme un contexte favorable pour entamer une transformation des schémas cognitifs des acteurs pour aller vers le développement d'une vision plus transversale de la chaîne logistique (transformation du Bâ intra-organisationnel).

L'ERP retenu fut celui d'un éditeur leader sur ce marché. Cet ERP présentait trois avantages majeurs au vu de la gestion de connaissances. Tout d'abord, proposer une grande richesse de processus adaptables à l'entreprise (pour capturer une partie des connaissances de l'entreprise en matière de processus), d'autre part une facilité de déploiement sur les différents sites du groupe et enfin une présence forte de cet ERP chez les clients (ces deux dernières caractéristiques favorisant la gestion ultérieure de connaissances inter-organisationnelles).

La première phase de déploiement d'un ERP consiste en la formalisation/adaptation du modèle de données et des processus standards de l'ERP aux processus métiers. L'équipe en charge du déploiement de l'ERP au sein de l'entreprise, en s'appuyant sur le fournisseur de la solution, propose une première formalisation des données et des processus standards de l'ERP qui doit être adaptée à la réalité des processus de l'entreprise. Ce contexte nous est apparu particulièrement favorable pour entamer une démarche d'apprentissage des acteurs de la fonction logistique en matière de processus métier. Aussi, nous avons choisi de prendre en charge cette phase et de lancer un travail de formalisation des processus métiers.

En tant que responsable de la fonction logistique, nous avons formalisé une première proposition de formalisation basée sur les référentiels métiers SCOR et ASLOG. Cette première formalisation des processus métier fut proposée aux 70 «utilisateurs finaux » de la fonction logistique interne à savoir, les acteurs du service logistique mais aussi les acteurs du service client (fonction commerciale). Grâce au travail en groupe des utilisateurs finaux la première formalisation à partir des standards ASLOG et SCOR a été enrichie. Elle a été confrontée à la modélisation des données et des processus standards proposée par le fournisseur de la solution ERP. A l'issue de cette confrontation, en plus de l'adaptation des processus standards de l'ERP permettant son implémentation chez BONFIGLIOLI, un processus supplémentaire «Gérer les commandes clients » a été formalisé. Le 
tout a permis de créer un document de formalisation des processus appelé « Modèle logistique de Bonfiglioli Transmission ». L'intérêt du partage et de la co-construction de ce document fut de permettre le cadrage des modes opératoires et idées. Ainsi chaque logisticien prit l'habitude de positionner son action ou problématique en fonction de ce modèle.

Après cette première étape de formalisation, pour poursuivre l'explicitation des connaissances des processus opérationnels de la fonction logistique chez Bonfiglioli Transmission, nous avons profité de la dynamique créée lors de la construction du modèle logistique de Bonfiglioli Transmission. Ainsi des réunions d'échange, ouvertes à tous les utilisateurs finaux Bonfiglioli Transmission (70 personnes), ont été organisées deux fois par semaine pendant 6 mois. Elles eurent beaucoup de succès. Nous fixions des thèmes autour des principaux processus logistiques et de leur mise en œuvre opérationnelle avec le déploiement de l'ERP. Ceci permettait de répondre à la majorité des questions. La plupart des questions étaient formulées ainsi « aujourd'hui je fais ça, demain je fais comment dans l'ERP ? » Les questions sans réponse ou dont les réponses étaient jugées insatisfaisantes étaient soumises aux Business Process Owner (BPO), dont la mission étaient de les valider et de les classer par niveau d'importance. La validation du processus se faisait systématiquement ensuite lors d'une réunion avec l'ensemble des personnes concernées par le processus pour comprendre et officialiser le processus intégral. Pour cela l'analyse de l'impact et de l'influence des différents acteurs et des enjeux de pouvoir dans les processus logistiques réalisée avec MACTOR nous a permis de constituer les groupes adaptés pour chaque processus à valider en mobilisant non seulement les acteurs mettant directement en œuvre le processus mais aussi ceux exerçant une influence importante sur leur bon déroulement. Pour chaque processus, l'implication de l'ensemble de ces acteurs a permis de créer un consensus autour de la formalisation de la connaissance et ainsi la création d'une connaissance partagée.

Toute cette démarche mise en place a permis de définir et d'ajouter 6 processus qui n'avaient pas été identifiés lors de la construction du modèle logistique de Bonfiglioli Transmission. 
En parallèle à cette formalisation des connaissances «processus », nous avons également entamé une démarche d'apprentissage construite autour d'un projet de professionnalisation des acteurs de la chaîne logistique. L'ensemble de la démarche de travail en groupe autour de la formalisation des processus et de leur nouvelle mise en œuvre avec l'aide de l'ERP nous est en effet rapidement apparu comme un support idéal pour former les collaborateurs de la fonction logistique de Bonfiglioli Transmission aux méthodes logistiques. Cette phase de formation était cruciale car elle allait permettre aux logisticiens d'associer leur apprentissage terrain à des techniques plus formalisées et plus efficaces. Au-delà, elle avait également pour but de permettre la poursuite de la formalisation des connaissances «processus ». L'intérêt des acteurs de l'organisation pour l'explicitation de leurs connaissances était susceptible de s'essouffler rapidement. Aussi est-il paru pertinent d'associer un apprentissage à l'explicitation. Les acteurs ne venaient plus seulement pour «donner» leurs connaissances mais aussi pour en apprendre de nouvelles. Ainsi la mise en place des données de base de l'ERP (fiche article, stratégie d'approvisionnement et de gestion des stocks, capacité poste de travail, nomenclatures, gammes, externalisation de phase de production, etc.) fut réalisée en groupes de travail entre utilisateurs finaux et donna lieu à explication et présentation de méthodes de gestion des processus logistique (MRP, Kanban...). Cette phase de structuration des processus, pourtant fastidieuse, fut ainsi acceptée et réalisée avec beaucoup d'enthousiasme : les logisticiens ont compris l'importance de leurs connaissances et que la formalisation de ces processus allait leur permettre une plus grande efficacité opérationnelle. En parallèle, les logisticiens, sans particulièrement s'en rendre compte, se professionnalisaient. A l'issue de ce travail en groupe, bien aidés et soutenus, certains logisticiens commencèrent à faire évoluer leur fonction vers plus de professionnalisme et d'efficacité. Par exemple ils devinrent plus exigeants et demandeurs d'une meilleure qualité de l'expression des besoins clients auprès du service commercial, condition d'amélioration du processus global.

\subsubsection{Transformer les schémas cognitifs pour développer une culture des réseaux, des chaînes,} du projet, du risque, du changement et de l'innovation

Comme nous l'avons souligné précédemment, s'appuyer sur l'introduction au sein de l'organisation d'un ERP nous a également paru pertinent pour enclencher une transformation des 
schémas cognitifs et développer une culture des réseaux et des chaînes. Toutefois, comme le précise Spalanzani (2003), pour optimiser la chaîne logistique grâce à la GC l'enjeu est de tirer partie de l'ensemble des spécificités des connaissances des acteurs de la chaîne logistique. Aussi, occulter les connaissances logistiques tacites d'urgence et de situation omniprésentes conduit à se priver d'un levier supplémentaire d'amélioration de l'agilité et de la performance de la chaîne logistique. C'est pourquoi en parallèle de la démarche évoquée précédemment, pour faire évoluer les schémas cognitifs et développer une culture des réseaux et des chaînes, il nous est paru adapté de mener une autre démarche moins centrée sur les connaissances «processus » et plus centrée sur le contexte partagé et les connaissances d'urgence et de situation des acteurs de la chaîne logistique (transformation du $\ll b \hat{a} »)$.

Les entretiens pour réaliser les cartographies des connaissances permirent une première explicitation de ces connaissances autres que «processus » mais aussi de nouer le dialogue, de comprendre la motivation, le parcours professionnel et parfois personnel de chaque collaborateur. Dès les premiers entretiens les collaborateurs se sont sentis écoutés et revalorisés. Par la suite, nous nous sommes donnés un guide de conduite simple (salutations quotidiennes, prise en considération des problématiques, des idées, etc.) pour ne pas perdre la qualité de ces contacts humains. Un premier climat de confiance et d'échange se créa. Pour le pérenniser et contribuer à une transformation des schémas cognitifs nous avons mené une action à deux niveaux.

Tout d'abord, lors des échanges quotidiens sur des problèmes quotidiens, nous rappelions régulièrement le lien entre tel problème organisationnel vécu au niveau individuel et tel autre problème afin de faire évoluer le cadre de pensée de résolution des problèmes d'un niveau individuel vers un niveau plus collectif et organisationnel. Rapidement cette dynamique a commencé à porter ces fruits. Un véritable changement de comportement a été constaté. Il est maintenant naturel de voir un préparateur de commande qui tient entre ses mains un composant mal référencé, aller à la réception pour partager ses connaissances et former la personne en charge de la réception afin d'éviter que ne se reproduise l'erreur. Alors qu'auparavant, le préparateur de commande aurait imputé la responsabilité du problème à une mauvaise gestion de la réception et l'aurait géré uniquement à son niveau 
individuel, il cherche maintenant à résoudre le problème par l'explicitation et le partage de ses connaissances en prenant en compte la logique organisationnelle.

Par ailleurs, nous avons également encouragé les collaborateurs à formaliser et partager leurs connaissances de situation et d'urgence sous la forme qui leur convenait le mieux. Initialement aucune structure de formalisation particulière n'a été proposée. Ceci afin de laisser les collaborateurs utiliser librement les outils à leur disposition (feuille de papier scannées, fichier excel, etc.). Progressivement, les acteurs de la chaîne logistique se sont sentis valorisés et ont commencé à proposer des méthodes à l'explicitation, la formalisation et au partage de leurs connaissances. Ainsi par exemple, un approvisionneur ayant été formé par ailleurs à la démarche qualité a spontanément proposé lors d'une réunion d'échange un document type de formalisation de ces connaissances de situation et d'urgence. Après avoir obtenu l'accord du département Qualité sur une telle démarche, nous avons accepté la proposition de l'approvisionneur. Lors d'une réunion d'échange suivante, ce document type fut expliqué et diffusé aux collaborateurs comme un modèle possible de formalisation des connaissances. Ce document fut approprié et utilisé par les 70 utilisateurs pour formaliser leurs connaissances de situation et d'urgence. Les documents produits par cette démarche ont été appelés « fiches modes opératoires ».

\subsubsection{Bilan de la phase thérapeutique}

Grâce à l'ensemble de cette intervention, en l'espace de trois mois les logisticiens ont officialisé 18 processus et 74 modes opératoires touchant les processus approvisionnements, gestion des commandes clients, déplacement des stocks, assemblage, livraison et retour. L'intervention a non seulement permis d'expliciter la connaissance «processus » et les best practices logistiques chez Bonfiglioli mais également a permis d'enrichir la connaissance « embarquée » dans l'ERP et son paramétrage, l'autre partie de la connaissance explicitée étant captée par les fiches modes opératoires. Elle a permis par ailleurs de créer une dynamique d'amélioration continue de la gestion des connaissances au service de l'efficacité de la chaîne logistique intra-organisationnelle ; sans oublier la reconnaissance et la professionnalisation des acteurs de la chaine logistique. 


\section{Mise en perspective de l'intervention}

Le SGC mis en place pour l'améliorer l'efficacité de la chaîne logistique intraorganisationnelle de Bonfiglioli Transmission se structure tout d'abord autour d'une formalisation régulière des connaissances «processus » embarquées au sein d'un ERP. Le SGC de cette manière remplit l'objectif d'explicitation et de formalisation des connaissances « processus » ouvrant la voie à une possible externalisation des connaissances susceptible de contribuer à une dynamique de développement des connaissances intra- mais aussi potentiellement inter-organisationnelles. Son utilisation permet au quotidien de disposer d'un référentiel de connaissances partagées par les acteurs de la chaîne logistique, potentiellement compatible avec un référentiel partagé inter-organisationnel. Au-delà, l'ERP remplit les deux contraintes fixées au TI support du SGC pour la chaîne logistique intra-organisationnelle à savoir la circulation en flux d'information et le choix de format compatibles avec les standards inter-organisationels. De fait, le déploiement de cet ERP chez Bonfiglioli Transmission s'inscrit dans un projet plus vaste de mise en place d'un ERP sur tous les sites Bonfiglioli et d'amélioration de la performance de toute la chaîne logistique inter-organisationnelle de Bonfiglioli par la GC.

Le SGC se structure aussi autour de fiches de description de modes opératoires permettant une gestion des connaissances de situation et d'action. Ce faisant, il s'adapte à une population qui n'est pas dans une culture de l'écrit mais plutôt dans une culture de l'action et de la résolution de problèmes dans l'urgence. Ce mode d'explicitation des connaissances individuelles d'urgence, de situation et d'action, très utile au quotidien, ouvre également la voie à une amélioration continue des connaissances « processus » gérées par l'ERP.

Enfin le SGC s'appuie sur une démarche de responsabilisation et de revalorisation des acteurs de la fonction logistique qui créée une dynamique d'apprentissage et permet une véritable transformation des schémas cognitifs pour développer une culture du projet, du risque, du changement et de l'innovation. Cette transformation du «Bâ » contribue également à la dynamique d'amélioration continue des connaissances «processus » et des connaissances formalisées via les fiches modes opératoires. La structure de ce SGC est synthétisée dans le schéma suivant (figure 2). 


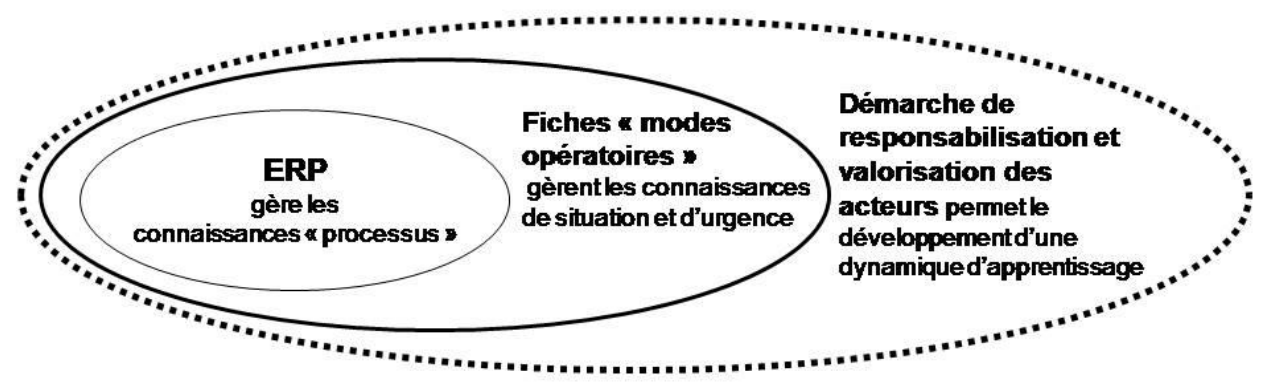

Figure 2. Le SGC pour la chaîne logistique intra-organisationnelle mis en place au sein de Bonfiglioli Transmission

Le SGC s'avère ainsi un dispositif permettant l'agilité et la flexibilité de la chaîne logistique au sein de Bonfiglioli Transmission. En situation d'urgence et d'action les acteurs développent des connaissances pour s'adapter aux changements internes comme aux évolutions de l'environnement extérieur à l'entreprise. Ces connaissances sont intégrées à l'organisation et à ces processus via le SGC. De la sorte le SGC devient un dispositif important pour l'adaptation de la chaîne logistique aux évolutions de l'environnement.

Ce travail met tout d'abord en évidence le rôle relativement faible joué par les TI en tant qu'outil de gestion des connaissances dans ce contexte. L'ERP en tant que tel n'est qu'une partie du SGC et ne gère que les connaissances formalisées. Son déploiement a été un prétexte à l'orchestration d'une démarche plus globale de responsabilisation et de valorisation des salariés permettant le développement d'une véritable dynamique d'apprentissage et la mise en place du SGC dans sa globalité. Ainsi dans ce cas, les TI sont surtout mobilisées en tant qu'outil de transformation des schémas cognitifs. Dans ce rôle, elles ont joué un rôle déterminant pour la conception du SGC chez 
Bonfiglioli. Elles agissent principalement comme un objet fédérateur permettant à chaque acteur de construire sa représentation de sa position, de ses pratiques métiers et de la position et des pratiques métiers des autres acteurs dans la chaîne logistique.

Cette intervention conduit à une deuxième observation autour du rôle déterminant de la prise en compte des zones d'influence et jeux de pouvoir pour la Gestion des Connaissances dans la chaîne logistique intra-organisationnelle. La caractérisation du rôle de ces zones d'influence et jeux de pouvoir dans la chaîne logistique a permis la reconnaissance du rôle de chacun des acteurs dans le partage des connaissances et a ainsi ouvert la voie à leur mise en confiance favorisant le développement de la dynamique d'apprentissage. Certes, en matière de GC, la motivation des acteurs et la conscience individuelle du pouvoir qu'ils détiennent de l'exploitation et de la détention de leur propre savoir est une donnée importante à prendre en compte pour développer des SGC efficaces. Toutefois l'importance des interfaces entre les différentes fonctions logistiques et les autres départements de l'entreprise semble renforcer la prise en compte de cet élément pour la conception de SGC adaptés à la chaîne logistique. La littérature insiste sur la difficulté de gérer ces interfaces qui sont considérées comme des lieux d'échange souvent conflictuels. Pourtant dépasser ces situations est reconnu comme primordial pour développer une « agilité logistique » (Christopher, 2000).

En termes d'implications managériales, ce cas d'intervention amène à penser que dans la fonction logistique la mise en place d'un Système de Gestion des Connaissances s'appuie moins sur une technologie que sur un accompagnement managérial. Tout l'enjeu est par la suite l'entretien de la dynamique d'apprentissage créée. Dans le cas Bonfiglioli étudié, ce problème apparaît moins comme un problème de management des Systèmes d'Information (comment faire pour que l'ERP soit bien paramétré et utilisé au quotidien par les acteurs ?) ou un problème de Gestion des Connaissances (comment faire pour que les connaissances soient toujours formalisées ?) que comme un problème managérial replaçant l'Homme au cœur des processus logistiques (comment entretenir la démarche de valorisation et de responsabilisation des acteurs ?). Au-delà, la mise en place de cette dynamique d'apprentissage et la transformation des schémas cognitifs associée a conduit les acteurs de la chaîne logistique à développer un regard transverse sur l'entreprise, une culture du projet, de la 
responsabilisation et de la prise en main des problèmes. Ceci pourrait conduire à terme à des problèmes de gestion de carrières ou à des problèmes de gestion opérationnelle des hommes. De fait, avec ce nouveau regard, les acteurs sont susceptibles d'identifier les problèmes et de les mettre en commun afin d'en comprendre leurs origines en recherchant le soutien et la reconnaissance de la hiérarchie quant à la résolution de ces problèmes. Cette transformation des schémas cognitifs des acteurs de la chaîne logistique va donc nécessiter une reconfiguration du rôle de la hiérarchie de cette fonction. Enfin les acteurs de la châne logistique ayant développé ce regard transverse, il va également falloir pour l'entreprise trouver de nouvelles voies d'évolution à ces acteurs. Il est vraisemblable qu'avec ce nouveau regard, les acteurs de la chaîne logistique se satisfassent plus difficilement de fonctions cloisonnées ou spécialisées. La définition des postes et la gestion de carrière de ces acteurs devra vraisemblablement tenir compte de cette nouvelle donnée.

Plus globalement, ce travail ouvre une réflexion sur les types de SGC à concevoir dans les organisations actuelles. De fait au sein de la chaîne logistique, l'objectif est de gérer des connaissances tacites, de situation et d'action tout en s'ouvrant à un contexte inter-organisationnel. La généralisation des entreprises en réseaux, l'aplanissement des hiérarchies, la gestion de situations de plus en plus dans l'urgence par les acteurs comme l'arrivée d'une nouvelle génération de salariés moins versée dans la culture de l'écrit fait que le contexte de la chaîne logistique pourrait être pris comme exemple pour les contextes de gestion des connaissances des entreprises dans les années à venir. Les conclusions du travail présenté dans cet article pourraient ainsi ouvrir des perspectives de réflexion pour la conception de SGC adaptés à ces nouveaux contextes.

En termes d'implications théoriques, ce travail confirme la portée des approches dites de la « réconciliation » pour la compréhension des problématiques de GC dans les organisations. Comme le souligne Felin et Hesterly (2007) et von Krogh (2009), le champ de recherche en GC a toujours été traversé par deux visions contradictoires sur l'origine de la création de connaissances et de la dynamique d'apprentissage dans les entreprises. Selon la perspective dite «collectiviste», la connaissance est fondamentalement un fait social au sens Durkheimien du terme. Ainsi l'approche fondée sur les ressources et des auteurs comme Kogut et Zander (1992) ou Teece et ali (1997) 
soutiennent cette idée que la connaissance naît de l'organisation et non de l'individu'. A l'inverse une perspective «individualiste » de la connaissance s'est développée, notamment en intégrant les travaux sur le «sensemaking»(Weick, 1995). Selon cette perspective, la création de connaissances dans l'entreprise repose d'abord sur les individus et leurs spécificités cognitives et c'est cette création de connaissance qui permet «l'organizing». Plus récemment, une perspective dite de la « réconciliation » (Felin et Hesterly, 2007) a vu le jour en GC autour des travaux sur la théorie de la pratique et la recherche en SI. Inspirés des travaux de Giddens (1984) et de Bourdieu (1972), ces travaux soulignent que la création de connaissances naît d'une interaction entre l'individu et l'organisation et que cette interaction nécessite la présence d'objets intermédiaires ou artefacts tels que la pratique de travail (Brown et Duguid, 2001) ou les TI (Alavi et Leidner, 2001 ; Orlikowski, 2002). Le cas d'intervention présenté dans cet article confirme le rôle déterminant que peut jouer l'artefact TI dans la construction d'une dynamique d'apprentissage faisant interagir individus et organisation. Il montre notamment comment le TI permet aux individus de se forger une compréhension commune de l'organisation, ouvrant la voie à la création de connaissances qui font elles-mêmes évoluer l'organisation. Ainsi ce cas confirme la pertinence de cette approche dite de la « réconciliation » et encourage à poursuivre les travaux en GC selon cette perspective.

Comme tous résultats de recherche-intervention, ce cadre de conception des SGC sera à déployer sur d'autres organisations pour en évaluer toute la portée. Toutefois ce travail permet d'enrichir les travaux sur la Gestion des Connaissances dans la chaîne logistique intraorganisationnelle. Au-delà il ouvre la voie à une réflexion plus théorique sur la pertinence des perspectives de recherche pour traiter de la problématique de la GC dans les entreprises.

\section{Remerciements}

Les auteurs souhaitent tout d'abord remercier l'entreprise Bonfiglioli Transmission et l'ensemble des acteurs de la fonction logistique pour leur implication et motivation dans la réalisation de ce projet de

\footnotetext{
1 "The firm has an ability to know independtly of its employees, or at least independtly of their conscious reasoning" (Spender, 1996, p.51)
} 
recherche. Les auteurs remercient également l'éditeur en chef et les évaluateurs anonymes de la revue SIM pour la richesse de leurs suggestions et commentaires sur ce travail.

\section{Références}

Akkermans H., van Helden K. (2002), "Vicious and virtuous cycles in ERP implementation: case study of interrelations between critical success factors", European Journal of Information Systems, vol. 11, p. 35-46

Alavi M., Leidner D. (2001), "Knowledge Management and Knowledge Management Systems: Conceptual Fondations and Research Issues”, MISQ, vol. 25, n¹, p 107-136, Mars 2001

Aviv I., Levy M., Hadar I. (2008), “Socio-Engineering Knowledge Audit Methodology (SEKAM) for Analyzing End-User Requirements", Actes de la conférence AMCIS, Toronto

Baskerville R.L., (1999) "Investigating Information Systems with Action Research", Communications of the Association for Information Systems (CAIS), vol.2, article 19

Binot C., Dudezert A. (2008), "Place du KM dans les nouveaux modes organisationnels : la network centric entreprise ", in Vers le KM 2.0, quel management des connaissances imaginer pour faire face aux défis futurs, ouvrage coordonné par A. Dudezert et I. Boughzala, Vuibert, p.151-167

Bourdieu P. (1972), Esquisse d'une théorie de la pratique: Précédé de trois études d'ethnologie kabyle, Librairie DROZ

Brown J.S., Duguid P. (2001), "Knowledge and organization: a social-practice perspective", Organization Science, vol. 12, p. 198-213

Camman C., Livolsi L. (2007) "Enjeux et difficultés de la gestion des cadres intermédiaires chez les prestatai-res logistiques ”, Logistique \& Management, vol 15, №2

Caron-Fasan M-L. et Farastier A. (2003), "Veille stratégique et gestion des connaissances ”, in Présent et Futurs des systèmes d'information, ouvrage coordonné par M-L Caron-Fasan et N. Lesca, Presses Universitaires de Grenoble, p. 237-264 
Chu ChenBing (2008), Leçon inaugurale de la Chaire Supply Chain Management, Ecole Centrale Paris, http://www.ecp.fr/fr/E_espace_entreprises/E4_chaires_entreprises/E4a_chaires_entreprises.ht $\underline{\text { m\#video }}$

Christopher M. Jüttner U. (2000), "Supply chain relationships: making the transition to closer integration”, International Journal of Logistics: Research and Applications, vol. 3, n 1, p. 5-23.

David A. (2000), "La recherche-intervention, cadre gé-néral pour la recherche en management", in Les nouvelles fondations des Sciences de Gestion, sous la coordination d'A. David, A.Hatchuel et R. Laufer, Vuibert, Collection FNEGE, p 193-215

Dudezert A. (2007), “Cartographie des connaissances et gestion des ressources humaines: exemple de l'ambiguïté cognitive des Systèmes de Gestion des Connaissances“, Systèmes d'Information et Management, vol. 12, n³, p. 31-56

Dudezert A. et Lancini A. (2006), "Performance et Gestion des Connaissances: Contribution à la construction d'un cadre d'analyse“, Actes des Journée des IAE, Congrès du cinquantenaire, Montpellier, France.

El Amrani R. (2008), "De l'intégration du système d'information à la vision transversale de l'organisation“, Systèmes d'information et Management, $\mathrm{N}^{\circ}: 4$ Volume 13, p.61-93,

Earl M.J. (2001), "Knowledge Management Strategies: toward a taxonomy“, Journal of Management Information Systems, vol.18, ${ }^{\circ} 1, \mathrm{p}$ 215-233.

Evrard Samuel K., Spalanzani A. (2009), “Apprentissage interorganisationnel et supply chain management : vers une gestion des connaissances partagée“, Actes du $14^{\text {ème }}$ colloque de l'Association Information et Management, Marrakech, Juin

Fabbe-Costes N. (2007), "La gestion des chaînes logistiques multi-acteurs : les dimensions organisationnelles d'une gestion lean et agile“, in La Gestion des chaînes logistiques multi-acteurs : perspectives stratégiques, ouvrage coordonné par G. Paché et A. Spalanzani, Presses Universitaires de Grenoble, Chapitre 1, p. 19-43 
Fabbe-Costes N. (2008), "Supply Chain Management et pratiques inter-organisationnelles de gestion des connaissances. Contribution à une réflexion sur le IKM (Inter-organiszational Knowledge Management “, in Vers le KM 2.0, quel management des connaissances imaginer pour faire face aux défis futurs, ouvrage coordonné par A. Dudezert et I. Boughzala, Vuibert, p. 93-121

Fabbe-Costes N., Lancini A. (2009), "Gestion inter-organisationnelle des connaissances et gestion des chaînes logistiques : enjeux, limites et défis“, Management et Avenir, n²4, p. 123-145

Felin T., Hesterly W.S. (2007), "The knowledge-based view, heterogeneity, and new value creation: philosophical considerations on the locus of knowledge", Academy of Management Review, vol. $32, \mathrm{n}^{\circ} 1, \mathrm{p} 195-218$

Gammelgaard B. et Larson P.D. (2001), "Logistics Skills and Competencies for Supply Chain Management “, Journal of Business Logistics, vol. 22, n², p. 27-50

Giddens, A., 1984, The constitution of society: Outline of the theory of structuration, Los Angeles/Berkeley: University of California Press

Godet M. (2006), Prospective stratégique : Problèmes et méthodes, Cahier du LIPSOR N²0

Gunasekaran A. et Ngai E.W.T. (2007), "Knowledge management in 21st century manufacturing“, International Journal of Production Research, Vol. 45, $\mathrm{N}^{\circ} 11$, p. 2391-2418

Hansen M.T., Nohria N., Tierney T. (1999), "What's Your Strategy for Managing Knowledge? “,Harvard Business Review, 77 (2), p 106-116.

Hult G.T., Ketchen D.J., Nichols E.L. (2003), “Organizational learning as a strategic resource in supply management“", Journal of Operations Management, $\mathrm{n}^{\circ}$ 21, p.541-556

Hult G.T., Ketchen D.J , Cavusgil S.T., Calantone R.J.(2006), "Knowledge as a strategic resource in supply chains“", Journal of Operations Management, $\mathrm{n}^{\circ} 24, \mathrm{p} .458-475$

Jones M.C., Cline M., Ryan S. (2006), "Exploring knowledge sharing in ERP implementation:an organizational culture framework", Decision Support Systems, vol. 41 p. 411 - 434

Kogut B., Zander U. (1992), "Knowledge of the firm, combinative capabilities and the replication of technology“, Organization Science, vol. 3, n 3, p. 383-397.

Lancini A. (2007), "Vers une gestion des connaissances dans les chaînes logistiques multiacteurs: état des lieux et perspectives“, in La Gestion des chaînes logistiques multi-acteurs : 
perspectives stratégiques, ouvrage coordonné par G. Paché et A. Spalanzani, Presses Universitaires de Grenoble, Chapitre 13, p. 233-246

Mathe H., Tixier D. (1980), La logistique, Que sais-je ? PUF

Nonaka I., Takeuchi H. (1995), The Knowledge-Creating Company, éditions Oxford University Press

Oppong S.A., Yen D.C, Merhout J.W.(2005), “A new strategy for harnessing knowledge management in e-commerce“, Technology in Society, vol. 27, p. 413-435

Orlikowski W.J. (2002), "Knowing in practice: enacting a collective capability in distributed organizing”, Organization Science, vol. 13, p 249-273

Pagell M. (2004), "Understanding the factors that enable and inhibit the integration of operations, purchasing and logistics", Journal of Operations Management, vol. 22, p 459-487

Reix R. (2005), Systèmes d'information et management des organisations, Vuilbert, 5ème édition.

Schultze U., Leidner D. (2002), "Studying Knowledge Management in Information Systems Research: Dis-courses and Theoretical Assumptions“, $M I S Q$, vol.26, n³, p 213-242

Sheffi, Y. and Peter K. (1997), "Logistics at Large: Jumping the Barriers of the Logistics Function“, James M. Masters, ed., Proceedings ofthe Twenty-Sixth Annual Transportation and Logistics Educators Conference, Council of Logistics Management, p. 1-26.

Spalanzani Alain (2003), "Evolution et perspectives de l'organisation et de la gestion industrielle : l'impact des systèmes d'information“", in Présent et Futurs des systèmes d'information, ouvrage coordonné par M-L Caron-Fasan et N. Lesca, Presses Universitaires de Grenoble, p. 19-44.

Spender J.C. (1996), "Making knowledge the basis of a dynamic theory of the firm", Strategy Management Journal, vol. 17 (Winter special issue), p. 45-62

Teece D.J., Pisano G.P., Shuen A. (1997), "Dynamic capabilities and strategic management”, Strategic Management Journal, vol. 18, p 509-534

Von Krogh G. (2009), "Individualist and collectivist perspectives on knowledge in organizations: implications for information systems research", Journal of Strategic Information Systems, vol. 18, p. 119-129 
Wei H-L., Wang E.TG., Pei-Hung (2005) "Understanding misalignment and cascading change of ERP im-plementation : a stage of view analysis“, European Journal of Information Systems, vol. 14, p.324-334

Weick K.E. (1995), Sensemaking in organizations, Sage Publications, Inc 